\title{
A Rather Accurate Piano Transcription of Pachelbel's Canon in D
}

\author{
Download links
}

Isaac David < isacdaavid@isacdaavid.info $>$

2017-12-06

Consider the following scenario: a musician wants to learn Johann Pachelbel's flagship composition, this time for solo keyboard. That is, learning it beyond picking its chords and phrases by ear. Maybe a friend of his insists on it being played on the church organ, as is customary for weddings. But to his disenchantment, the pianist tragically resolves that all of the existing adaptations are way too uncanonical. How come?!

\section{About Canon}

Allow me to explain with a brief refresher what I mean by uncanonical, and why that could be a bad thing. In music, a canon refers to a certain kind of polyphonic texture whereby the initial melody's pitch and duration relationships (let both be denoted by "canonical melody" or "canon proper") are clearly and orderly restated by subsequent voices - truly a staple of imitative counterpoint. Short canons akin to repeat ad nauseam in coping for their limited duration abound in folk music (these are more properly called "rounds" though). Whenever the canon texture features prominently throughout a piece the latter may well be named after it, regardless of its musical form. $\underline{2}$. Hence the reification of titles like "Bach's Goldberg Canons" or "Brahm's 13 Canons for Female Chorus".

Let $\mathbf{v}$ - a voice's waveform - be a function of time $(\mathbf{v}: \mathbb{R} \rightarrow \mathbb{R})$. We can think of any textured piece as the higher-order function $\left((\mathbb{R} \rightarrow \mathbb{R})^{N} \rightarrow(\mathbb{R} \rightarrow \mathbb{R}) ; N \in \mathbb{N}\right)$ that sums voices point-wisely. This is no less true of canons:

$$
\mathbf{c}\left(\mathbf{v}_{0}, \ldots, \mathbf{v}_{N}\right)=\sum_{n=0}^{N} \mathbf{v}_{n}
$$

There's a so-called "perfect canon" which follows this layout to a hair's breadth, and does it reject transformations from voice to voice. In doing so it sets the standard not just for relative pitch and

\footnotetext{
${ }^{1}$ It's interesting to note that contrapuntal textures tend to override other common-practice-period naming conventions; perhaps because explicitly polyphonic compositions are rarer than — and hardly absent of — form.

${ }^{2}$ I'll be reserving the noun "form" for its musical meaning, which has close connections to formal grammar insofar as temporal structure is concerned; although there certainly can be some form to musical texture/simultaneous events. It's called geometry elsewhere.
} 
rhythm, but also for their absolute counterparts. Loudness and timbre (and perhaps articulation) are the only qualities that may be spared from this equation.

Let's now go to the finer cross-sectional level to reveal some structure. $c:\left((\mathbb{R} \rightarrow \mathbb{R})^{N} \rightarrow \mathbb{R}\right) \rightarrow \mathbb{R}$ is the value of $\mathbf{c}$ at any given instant $t$ :

$$
c\left(\mathbf{v}_{0}, \ldots, \mathbf{v}_{N}, t\right)=\sum_{n=0}^{N} \mathbf{v}_{n}(t)
$$

For the strictest conceivable canons there's a reference $\mathbf{v}_{0}$ such that $\mathbf{v}_{n}(t)=\mathbf{v}_{0}(t-n \tau) ; \tau \in \mathbb{R}$, where $\tau$ is the inter-voice period: 3

$$
\vdash \mathbf{c}\left(\mathbf{v}_{0}, \ldots, \mathbf{v}_{N}\right)=\mathbf{c}\left(\mathbf{v}_{0}, N, \tau\right)=\sum_{n=0}^{N} \mathbf{v}_{0}(t-n \tau)
$$

Canon in D from the two-part "Canone e giga per 3 violini e basso" (Canon and Gigue for 3 Violins and Bass) is one such perfect canon in three voices for some five minutes or more - the whole movement indeed - at a rather high note density. At this point you are supposed to have taken a bow before the man; but just in case that didn't occur, ponder how our canon proper is always introducing uncharted melodic territory: there's little overarching structure to the piece and therefore nothing like big repetitions. $\underline{4}$ Every two bars in the melody form a statement, and the furthest you get to grouping them, for the most part, is in adjacent pairs like this: $\operatorname{aAbBcCdD} \ldots .5$

Let that sink in for a moment. How on earth do you keep tacking fresh, yet coherent, melody onto the stream, while also making sure it fits harmonically with what you just set in stone two and four measures ago? Would you just keep looking $\tau=2$ measures and $2 \tau$ back to decide what the next note should be? Not this time. Hold your horses counterpoint junkies!, because the knee-jerk answer won’t help you here either. Pachelbel provides a more graceful solution which I think hasn't been celebrated enough. You've got to think more like a jazz soloist this time.

Could the implied semi-shared two-bar windows be enough to warrant harmony?:

\footnotetext{
${ }^{3}$ In the limit when $n \rightarrow \infty$ and $\Delta \tau \rightarrow 0$, the perfect canon is a special case of a convolution, of $\mathbf{v}_{0}$ and a per-voice amplitude scaling function (constant 1 for equally loud voices). Id est:

$$
\int_{-\infty}^{\infty} \mathbf{v}_{0}(t-\tau) d \tau
$$

${ }^{4}$ Most people can't help notice an intro, a middle part and an end stemming from an overall rise and decline in rhythm; but they are quite independent from one another. The music seems to proceed by very shortsighted Markovian dynamics. However, no autocorrelation analysis was performed.

${ }^{5}$ Kathryn Welter, "Johann Pachelbel: Organist, Teacher, Composer: A Critical Reexamination of His Life, Works, and Historical Significance", PhD diss. (Cambridge, MA: Harvard University, 1998): pp. 207-208.
} 


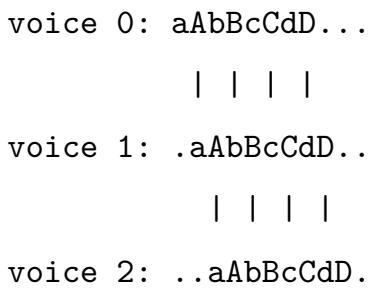

Admittedly, last time you listened to it neither were $1 / 3 \mathrm{rd}$ of the notes perpetually guilty of arbitrary dissonance (although the actual contrapuntal freedom does contribute to some wild extended harmonies, which I think wouldn't be there otherwise), nor was the melody dull and rigged. It is true that the canonical melody was clearly meant to retreat and yield the lead to voice two or three; in places such as bar 13 and 14, 23 to 26 and 39 to 42 - this produces a sensation of settlement in preparation for the next big pseudo-section - but nowhere else is it less than co-protagonist. In fact, this composition is famous for the exact opposite qualities: it's conspicuously squarish in its harmonic development, and exuberant in melodic contrasts by and large. So a bar pipeline modulo four won't get you all the way there.

Congratulations to those who pursued the idea to the point of inferring that a chord cycle at the finer statement level would work just fine. An obstinate ground bass (only the base notes are shown in score) can be heard on its own before the violins come in, and accompanies them throughout each statement; $\underline{6}$ so that the canonical melody is free to roam the frequency spectrum under its supervision. As long as new incursions stand in some relationship to the escort chords, they're guaranteed to also stand in some sensible relationship to whatever pitch was played around $2 n$ full measures ago, by virtue of the chord circle (I-V-vi-iii-IV-I-IV-V). One could argue this is along the lines of a cantus firmus, but the actual lesson to be learned here is that Canon in $D$ is as much a chaconne as it is a canon: canon by its texture, chaconne by its passacaglia form with the thematic material on the bass.

\section{No transcriptions available}

All that said, one would imagine that faithful transcriptions would have already been made for keyboard instruments, or at least a nice reduction that preserves the essence of the variations and canonical texture after which the music was conceived. This is the one essence you want to preserve for it to be called a transcription! Here pedaled organs have an enormous advantage relieving the left hand from playing ostinato; and although I will be focusing on the much more widely available piano configuration,

\footnotetext{
${ }^{6}$ Kathryn Welter, "Johann Pachelbel: Organist, Teacher, Composer: A Critical Reexamination of His Life, Works, and Historical Significance", PhD diss. (Cambridge, MA: Harvard University, 1998): pp. 207-208.
} 
my brief survey of IMSLP 7 suggests that existing transcriptions don't take this instrument to its full potential. Jeffrey Cooper's offer (2015) omits a few notes and one of the 32nd-note statements in full, and for reasons unknown to me doesn't take the opportunity to transfer voices verbatim (insofar as human hands would permit) to the score, independent beams and stems included. Other than that, it's an accurate transcription. It shouldn't take much effort to distill polyphony out of its seemingly atomic chords.

Meanwhile in piano land we find like a dozen arrangements - many under the misleading guise of transcription - all with varying degrees of outright artistic license. Paul Barton has conveniently recorded many of them, should you like to asses my claim. Just don't get too used to the slow tempi because those are apocryphal too. Despite all the popularity after decades of piano recordings for massive consumption, I tremble not to say that nobody knows what Canon in D sounds like anymore.

Some of the most down-to-earth arrangements are too simple to be of more than pedagogic value. Lee Galloway's (2000) fast modern accompaniment seems popular; but formally speaking this arrangement is only a glimmer of the original piece. The drive for arpeggiated ground bass may have originated very early with Sergei Lyapunov's posthumous? transcription. This I could not date, but I infer it must have been written between 1919 (when Canon in D was first rediscovered by Gustav Beckmann뽀) and before Lyapunov's death in 1924. It's a nice arrangement overall, notwithstanding the sacrificial fate that part of the polyphony and a few harmonies undergo to make room for the nonstandard accompaniment. H.M.'s variant (2007) comes close to being a nice satisfactory and accessible reduction if not for the fact that it omits entire statements. Don Simons' (2011) transcription for harpsichord is a solid canon in two voices. This approach on one hand bestows accessibility to the most unfathomable passages; however I also think that a strict two-voice transcription isn't being ambitious enough, and those boring (and frankly unrecognizable) parts at the other side of the coin are testament to it, for they were meant for voice three to shine. It's great middle-ground nonetheless; you could learn Don Simons' rather observant reduction before attempting to raise the bar.

Now pipe misconstructions down. I have nothing against wondrous re-imaginations; quite on the contrary: I'm happy that decades of propaganda and outrageous illiberal policies put forth by Big Copyright haven't quite killed the very same cultural process that nourished all the great artists of the past. $\underline{9}$ But in the same vein there must also be a place to purist renditions, historically informed performances and all that stuff; and it's a shame that such an iconic piece cannot be enjoyed in full for

\footnotetext{
${ }^{7}$ International Music Score Library Project, also known as the Petrucci Music Library

${ }^{8}$ Gustav Beckmann, "Johann Pachelbel als Kammerkomponist", Archiv für Musikwissenschaft 1 (1918-19): 267-74. The Canon is found on p. 271. http://www.archive.org/stream/ArchivFrMusikwissenschaft01191819\#page/n293/mode/2up

${ }^{9}$ Search "Copyright vs Community" by Richard Stallman online and watch any of his lectures if this sounds alien to you. They are available in English, French and Spanish.
} 
the most iconic of all polyphonic instruments. Or can it? Well, grieve no more, because I've decided to put an end to such a cosmic travesty. You are about to join me in a journey to establish the canonical Canon for piano.

\section{How the sauce was made}

I'm a GNU Lilypond guy as well as a libre culture advocate, so I was pleased to discover that the nice folks at Mutopia had already typeset a standard score in a very extensible Lilypond project released under a free license. I'm indebted to them. In spite of this legacy, it took several weeks of manual adjustments to come up with a transcription I would deem worthy of sharing.

For one, the four-part score had to be merged in the two-staff kind of system pianists are familiar with. In the absence of a trustworthy piano transcription I first learned to play Canon from a full score, presuming that it would be more cost-effective than making the transcription. I was wrong, and the ensuing boondoggle is what motivated me to sign up for this task. I don't recommend reading from the ensemble score to any performer of solo polyphonic instruments, except for analysis, or as an exercise for improving your cephalopodan craft of decoupling fingers from hands, also known as voicing. Voices converge every now and then, just to jump afterwards from register to register more often than not, making the reading of staves which don't respond to these changes tantamount to untying a really messed-up knot. This is illustrated in Figure 1, an excerpt corresponding to bar 36.

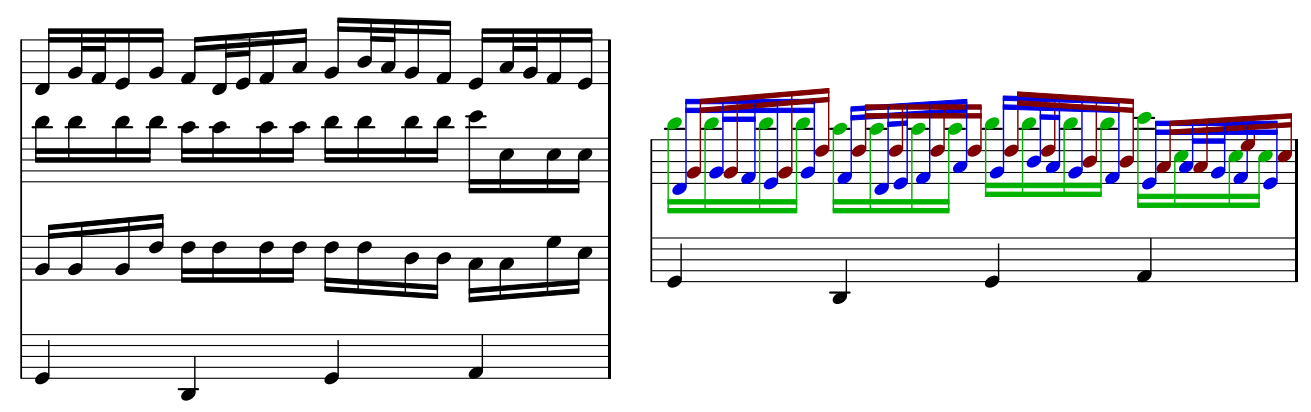

Figure 1: As comical as the version on the right might look, it's already a clear winner when it comes to encoding the information we are interested in; such as the fact that voice one is occupying the lower register, or that voice two and three briefly interchange positions near the end, or that note heads get duplicated on three occasions. Sources $\underline{1}$ and $\underline{2}$.

To aid with learning the demanding fingering I have decided to reserve each staff to a hand. $\underline{10}$ This comes at the expense of a slightly less appealing engraving and lots of ledger lines, but then again, accurate sound is our goal and the waving of your fingers is reduced to a means towards that end. With music so arcane to keyboard technique I'd rather convey hand position in the most straightforward

\footnotetext{
${ }^{10}$ Except for exactly five properly annotated wide arpeggios whose ends I play with the left hand.
} 
way possible.
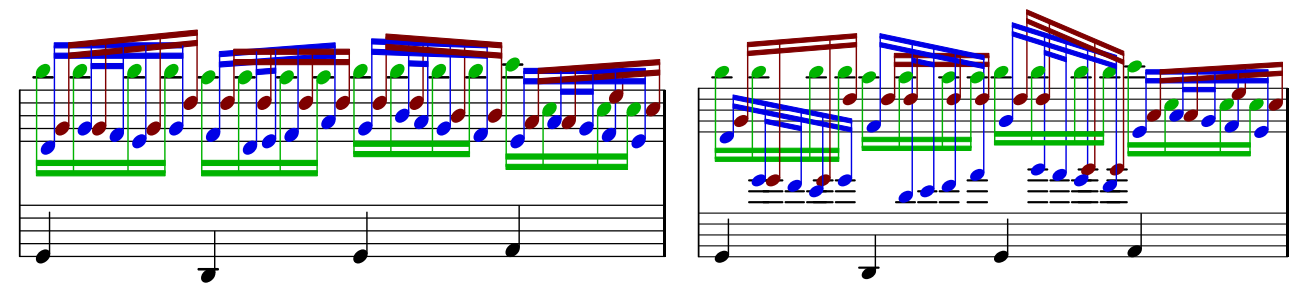

Figure 2: Keyboardists, like many other kinds of musicians, know that half their profession is a struggle for ergonomics. Fingering is suggested from the very choice of staff, but also clarified using numbering during more ambiguous passages. Sources $\underline{2}$ and $\underline{3}$.

Migrating to a piano staff was just the initiation in a long path towards purification. Not even mighty Lilypond was able to cram three unhinged, beam-full voices without leaving some collisions along the way. OK... many collisions. To its credit, any other engraving program would have probably scored worse. Gone is the elegant source code that so wittingly exploited the inherent structural redundancies.
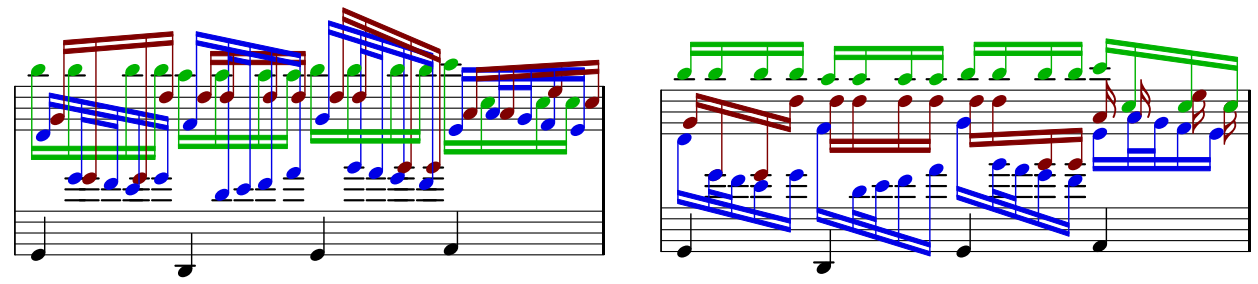

Figure 3: Collisions at bar 36 before and after manual directioning. Sources $\underline{3}$ and $\underline{4}$.

Notice how duplicate noteheads have been merged, and so will be their colors. À propos des couleurs: they are present in the final score, but you could easily change them from the file color.ily. 11 I think this is a much better system for voice bookkeeping than the standard notation. Having angled connectors all over the place, competing for perception with note stems and beams, is what I like to call noise. I'm told that the additive primary triad/vector basis is the safest for our $>8 \%$ of colorblind population. That it lends itself so naturally to the idea of $\left(\begin{array}{l}3 \\ 2\end{array}\right)$ possible superpositions is a happy coincidence. Lucky us, we never ran into a triplicate note.

\footnotetext{
${ }^{11}$ Or just print the provided score in black ink to still get some of the contrast in gray scale.
} 

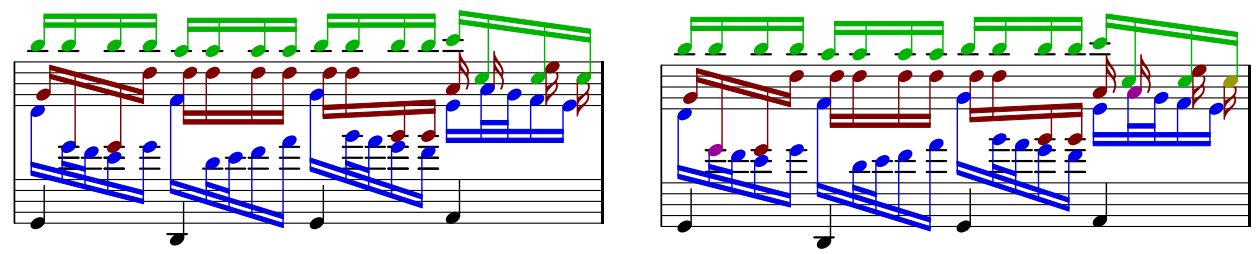

Figure 4: Sources $\underline{4}$ and $\underline{5}$.

Ideally we would stop at this point. If we did, the score would be completely accurate but playable by no one. This is no piano music. Rachmaninov was reportedly able to play rich chords that stretched a 13th with a single hand, $\underline{12}$ but these would have been either isolated/slow or repeating chords. Parallel-ish harmonies in very rigid species counterpoint at best (just like every other chord-heavy passage). Under no circumstances would you expect a freak to be able to handle chords of that nature unexpectedly bubbling as a happenstance of a composite-rhythmic, free-counterpoint polyphony. Well, I counted at least eight open triads that would require you to span a 13th with one hand in this piece. Evidently, some adaptation is due in order to cope for these and other quirks of solo piano music, if we are to fancy ourselves as solo piano music.

Affected notes can be either omitted or displaced horizontally or vertically. There's no need to do the former since a non-leading note could be always moved an octave, or the whole chord broken up into an arpeggio for only a fraction of the cost that full omission entails. We won't get into harmonics analysis to actually compare sounds, but it seems to me that in general not only is an octave which doesn't step over the melody less psycoacoustically different than no note at all. It's also the cumulative harmonic effect that is best survived by the same chord in a different inversion or "more closed" position. With the main melodic parts and the chord roots in place, everything else is relatively free to jump octaves.

Problematic notes are therefore roughly dealt with according to the following heuristics:

0. Use the sustain pedal at discretion to try to respect note lengths, even if it means prolonging other notes. Once every quarter note (every harmonic change) starting from measure 9 works well for me keeping blur levels low.

1. Try breaking voices into an arpeggio, consider including the bass, and see if that solves anything.

2. Could the passage be otherwise manageable had there been no ground bass note at that beat, delay the latter by an epsilon, as though it were an acciaccatura.

3. If that doesn't rock the boat and harmonic fodder is available, transpose it to a better octave and mark the original pitch with a dead note (so that the modification is just as noticeable and

\footnotetext{
${ }^{12}$ D. A. B. Young. "Rachmaninov and Marfan's syndrome". British Medical Journal, vol. 293: p. 1624. (1986). https://www.ncbi.nlm.nih.gov/pmc/articles/PMC1351877/pdf/bmjcred00266-0038.pdf
} 
as potentially reversible as the arpeggio symbol).

4. Contrived still? Repeat now that the chord has changed.
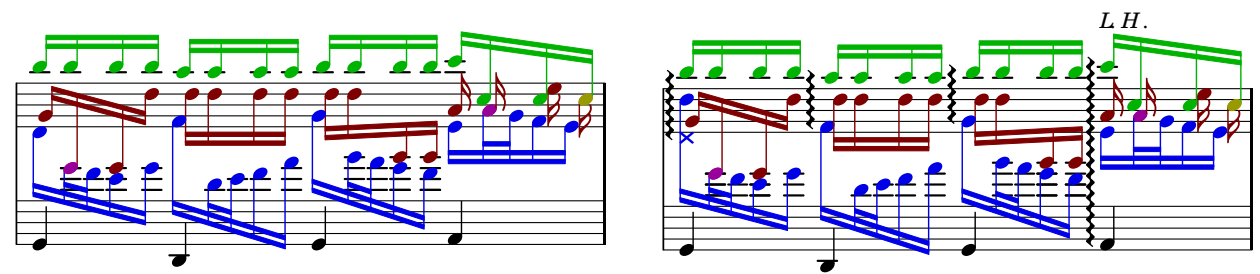

Figure 5: Final adjustments at bar 36, geared towards rendering the score playable on an average keyboard, by the average adult hand. Sources $\underline{5}$ and $\underline{6}$.

Ready for showcase it is. Some parts still come accross as challenging in excess because of our selfimposed modifications quota (and I have been playing it for well over a year). The following demo was recorded in three different sessions to keep the number of errors low, but keep in mind I'm by no means a professional. The last thing I want to do is paint a picture too dark that it will drive potential performers away. Don't let the music sheet daunt you either: baroque music looks more intimidating than it really is when you aren't used to seeing so much clutter on paper. 